Published online 2017 April 13.

Abstract

\title{
Clinical Implication of Intra-Amniotic Sludge in Predicting of Preterm Delivery
}

\author{
Narges Afzali, ${ }^{1, *}$ and Fatemeh Baghalsafa ${ }^{1}$ \\ ${ }^{1}$ Islamic Azad University Mashhad Branch, Mashhad, Iran \\ "Corresponding author: Narges Afzali. E-mail: nafzali2007@yahoo.com
}

Received 2016 December 21; Accepted 2017 February 08.

\begin{abstract}
Background: Preterm labor is defined as labor occurring prior to 37 weeks which .There are different factors which can lead to preterm labor including maternal causes, chorioamnionitis, cervical insufficiency and others. Because there is no satisfactory clinical method fop prediction of preterm labor, so it is important to look for some probable predicting markers by para clinical investigation.

Objectives: The main goal of this study is evaluation of relationship of intra-amniotic sludge in ultrasound exam and preterm delivery.

Methods: In a cohort study included 84 pregnant women in 18 - 32 weeks of gestational age referred to Islamic Azad university of mashhad hospitals in 2015. Cervical length measurement and intra-amniotic sludge were examined by transvaginal probe. Depending on sludge density the cases were divided to three groups: no amniotic sludge, light sludge and dense sludge. According to gestational age at delivery time the women were classified into two groups: term (delivery after 37 weeks) and preterm (delivery before 37 weeks).

Results: The prevalence of preterm delivery was $14 / 3 \%$. The frequencies of term delivery in mothers with dense, light and no sludge were $78 / 6 \%, 82 / 1 \%$ and $96 / 4 \%$ respectively, and for preterm delivery they were $21 / 4 \%, 17 / 9 \%$ and $3 / 6 \%$ respectively. But there was no statistically significant relationship between term and preterm delivery and type of sludge. Neonatal weight in mothers with sludge, was significantly lower than mothers without sludge.

Conclusions: There is no statistically significant relationship between preterm delivery and type of sludge.
\end{abstract}

Keywords: Intra-Amniotic Sludge, Transvaginal Ultrasound, Preterm Birth, Cervical Length

This is an abstract presented in the 33rd Iranian congress of radiology (ICR) and the 15th congress of Iranian radiographic science association (IRSA). 